\title{
MODEL EQUATIONS AND FORMATION OF STRUCTURES IN MEDIA WITH MEMORY
}

\author{
A.S. MAKARENKO
}

PACS 02.10.Kn, 02.20.Uw

(C) 2012

Institute for Applied System Analysis at National Technical University of Ukraine "KPI"

(37, Prosp. Peremogy, Kyiv 03056, Ukraine; e-mail: makalex@i.com. ua)

\begin{abstract}
We propose new types of models of the appearance of small- and large-scale structures in media with memory, including a hyperbolic modification of the Navier-Stokes equations and a class of dynamical low-dimensional models with memory effects. On the basis of computer modeling, the formation of the small-scale structures and collapses and the appearance of new chaotic solutions are demonstrated. Possibilities of the application of some proposed models to the description of the burst-type processes and collapses on the Sun are discussed.
\end{abstract}

\section{Introduction}

The basic equations for heat transfer and hydrodynamics are usually parabolic heat equations and the NavierStokes hydrodynamic equations. But these equations lose their applicability in extended media, when the characteristic scales of the variation of parameters are less than the correlation time and the correlation length (relaxation or memory and nonlocality effects). Many examples of nonapplicability were found in turbulence. Therefore, more correct equations should be used in such cases. As for the description of transport processes, we mentioned some well-established results in the theoretical physics.

The first famous idea is the existence of a hierarchy of description levels. If there are $N \gg 1$ particles, we have many levels for description: $N$ deterministic dynamical Newton laws for the movement of particles; Liouville equation for the $N$-particle distribution function, Boltzmann equation for the one-particle distribution function, hydrodynamic equations for macroparameters (NavierStokes equations), and thermodynamic equations. The choice of a level of description depends on the degree of a deviation from the equilibrium.

The second important idea is the existence of many interrelating relaxation processes and many time and space scales with different relaxation times and lengths. The memory and nonlocality effects are common for all levels. Each level of description has its own specific type of chaos, autowave solutions, collapses, and so on. It should be stressed that each level of description has its own model equations with typical behavior of a solution. The problem of defining the typical chaotic behavior for a given level is especially interesting.

The turbulence is a bright example of such complex phenomena. It is well known that turbulence is encountered very often in broad classes of natural processes. It is widely recognized that turbulence is a strongly nonequilibrium phenomenon. Investigators dealing with it in the specific branches of science, as a rule, formulate the essence of turbulence on the intuitive level. This results in the lack of a generally accepted and unified definition of turbulence. In specific cases, one can encounter different types of turbulence. This difference is fixed, for example, in the plasma theory (strong and weak turbulence).

Till now, the definition of turbulence, which would be strict and evident in all cases, is absent. To imagine a set of possibilities in this question, we will recall a few descriptions of turbulence mainly related to hydrodynamics.

"Turbulent motion of fluid at large enough values of the Reynolds number is characterized by the extraordinarily irregular, random change of the speed in time at every point of a stream (the developed turbulence). The speed always pulsates near the some mean value" [1].

"Turbulent motion is, generally speaking, vortical" [1].

"So, the turbulence is a vortical flow of a viscous fluid which is stochastic in the sense that it is characterized by: 1) sensitive dependence on the initial conditions (this caused by the exponential divergence of the initially close phase trajectories); 2) all phase trajectories are dense almost everywhere; thus, any initial nonequilibrium distributions of probability in the phase space tend to the limiting equilibrium distribution; 3) mixing in the phase space (and, as a result, ergodicity, by the rapid fading of temporal correlation functions and the continuity of frequency spectra); 4) the developed turbulence possesses additionally multimodes and, as a consequence, manifests the chaos in its spatial structure at any fixed moment of time" [2]. 
"This motion is so complicated and so not studied enough, that is is difficult to answer the questions: what is turbulence, and which kinds of basic features of turbulent motion are?" [3].

"Ones of the most considerable features of turbulence are the co-operation of order and accident... The accident in turbulence is related to the extreme sensitiveness to disturbances. At the same time, the chosen statistics is roughly stable at disturbances" [4].

"What is turbulence? Some light for this difficult purpose is explained by the study of offensive turbulence or weak turbulence which is an aspect of chaos" [5].

"The following definitions of turbulence can be offered... A turbulent flow should be unpredictable in the sense that a small uncertainty at the initial time will grow so that the strict deterministic prediction of its evolution is not possible; second, a turbulent flow should satisfy the property of the increasing mixing" [6].

Still, a number of determinations of the theory of plasma turbulence are presented that, as will be argued below, has definite interest. As for hydrodynamic turbulence, the most common descriptive determination is: "Any state of plasma with the strongly developed noises and vibrations is accepted as the turbulent one" [7].

The selection of collective degrees of freedom in plasma turbulence and the construction of turbulence on their basis are of importance.

"Presentation which is usually inlaid in the notion of turbulence substantially differs from the notion of molecular motion. Speaking, for example, about the motion of any element of fluid, the motion of some macroscopic volume is implied, containing the large number of molecules... In other words, the hydrodynamic motion of fluid corresponds to the definite collective degrees of freedom... It is possible to define the concept of "turbulence": the turbulent motion of macroscopic bodies is such a motion, in which the collective degrees of freedom are intensively excited and carried an accidental character" [8].

As a rule, it is assumed that turbulences in plasma and in fluid are different. In [9], the comparison of turbulence in plasma with turbulence in incompressible fluid is given. It turns out that the instability of fluid causes the excitation of whirlwinds, and there are difficulties with the flows of energy. It is considered that the indicated difficulties have a basic character and are connected with the strong turbulence in incompressible fluid. This is expressed by the following: whirlwinds generally do not have their own frequencies, and the time of transmission of energy from one whirlwind to a neighboring one is about one turn of the whirlwind.
Unlike the motion in incompressible fluids, there are a lot of collective oscillating motions in plasma which have their own definite frequencies. The time of transmission of energy of these vibrations to the neighboring scales (or neighboring wave numbers) can essentially exceed the characteristic period of vibrations $\omega_{k}^{-1}$. Because of this "elasticity" of collective motions, a small parameter $\omega=$ $\tau \omega_{k}^{-1} \ll 1$ can appear. It is considered that the presence of this small parameter allows one to use the regular methods of decomposition of the energy of turbulence and to build the theory of weak turbulence [9]. The role of dispersion for plasma turbulence is underlined here.

So, in [10], it is indicated that the dispersion is absent in incompressible fluids. Therefore, in this situation, we see the turbulence of whirlwinds and the transmission of energy between them. Plasma possesses many excitations with dispersion, co-operation, and weak turbulence [10]. In addition, there is the strong turbulence of solitons in plasma. Let us note that the results of investigations presented in the cited work presumably show that a sharp barrier between the types of turbulence in plasma and fluid is not present, and the situation turns out to be more difficult and interesting. Plenty of determinations indicates that the common determination of turbulence is absent, and there can be one specific type of turbulence in every specific process. This distinction is fixed, for example, in the theory of plasma (strong and weak turbulence).

But even if a rigorous definition exists, we must use some mathematical idealization in the theoretical study of this complicated phenomenon. For distributed objects, we usually take the nonlinear partial differential equations (PDEs) or integro-differential equations. Then mathematical models of the stochasticity connected with the unstability in systems are investigated.

The mathematical object on the hydrodynamic level is the system of PDEs for the velocity, pressure, and temperature. Such a system includes equations of motion, as well as the constitutive equations, which give the expressions for the dependence of deformations on stresses [11]. The instant connections between displacements and stresses lead to, in the case of the incompressible fluid, the Navier-Stokes equations. The memory effects take on the form of integral constitutive equations. The simplest exponential kernels lead to the constitutive equations introduced formerly for viscoelastic media. In such cases, we obtain the simplest modification of the NavierStokes equations, namely, the so-called equations for the Maxwell media ([12-14]).

Such modified system [12-14] is still a very complex mathematical object. In order to understand its proper- 
ties, one can investigate the simpler model equations. It is well known that such a model equation for the Navier-Stokes system is the nonlinear Burgers equation one-dimensional in the spatial variable [9]. This equation was introduced empirically, and it was later derived within the asymptotic approach. It has been recently proposed [10] to include a second-order derivative with respect to time in the Burgers equation. The main goal of such a modification was the incorporation of memory effects. Then we have the model equation, the socalled hyperbolic modification of the Burgers equation one-dimensional in the spatial variable $[15,16]$.

Its numerical and analytical studies reveal many curious properties. One of the most interesting properties is the existence of blow-up (collapse) solutions. However, not long ago, the two- and three-dimensional model equations for viscoelastic systems did not exist. Following the lines of deriving the Burgers equation, we put forward multidimensional model equations. The analysis of the full system [12-14] and the physical properties of systems with memory (fast heat processes, plasma, turbulence, statistical mechanics, viscoelasticity) allows us to pick out some necessary properties that should be accounted for by model equations. Such properties are viscosity, mass transfer by convection, and a finite speed of disturbances propagation. In addition, the properties of such model equations should resemble, in some specific cases, the behavior of original equations [12-14] and, what is more important, the behavior of real objects (for example, the stability properties). Our analysis leads to some new model equations with such properties. The simplest two- and three-dimensional equations have the form

$\mu \frac{\partial^{2} \mathbf{u}}{\partial t^{2}}+\frac{\partial \mathbf{u}}{\partial t}+u_{k} \frac{\partial \mathbf{u}}{\partial x_{k}}=c \Delta \mathbf{u}$

The nonlocality may be incorporated in the model equations as well.

\section{The Choice of Model Equations}

Since a deep theoretical description of mathematical models for hydrodynamic processes can be carried out by means of the methods of theoretical physics, we describe very briefly some main concepts from statistical physics relevant to the choice of model equations.

Begining from the works of N. Bogolyubov, M. Born, H. Green, J. Kirkwood, and J. Yvon (BBGKY), the canonical approach in the theoretical physics is as follows. Let us consider the medium constituted from $N$ independent particles. Then, in classical physics, we can describe the particle movement precisely by a system of ordinary differential equations (the Newton equations). But the statistical physics considers the ensemble of the system by introducing distribution function $f_{N}\left(x_{1}, x_{2}, \ldots, x_{N} ; t\right)$ for the probability distribution of particles at time $t$. The function $f_{N}$ is evaluated from the Liouville equation

$$
\frac{\partial f_{N}}{\partial t}+\left\{f_{N}, H\right\}=0
$$

where $H$ is the Hamiltonian of the system, and $\{\cdot\}$ are the Poisson brackets.

However, the function $f_{N}$ is too informative for the description of hydrodynamic phenomena. Usually, all necessary parameters are macroparameters (for example, the temperature, pressure, and velocity: $T, P$, and $V)$. The main leading principle in such a case is the reduction of the collection of description parameters. The reduction procedure deals with some hierarchical levels. First, by integrating over some variables in the phase space, we can go to the one-particle distribution function $f_{1}$ with the BBGKY chain of equations for $s$-particle distribution functions. Note that, for $f_{1}$, we can obtain the well-known Boltzmann equation. These stages with distribution functions are named kinetic.

The further averaging with the one-particle distribution function leads to macroparameters $T, U$, and $P$. Usual procedures lead to well-known equations of the hydrodynamic type: the parabolic heat equation, the Navier-Stokes equations, and so on. But a more correct description leads to more complicated equations with memory effects. The reason for the origin of memory effects under reduction processes is very well described in theoretical physics, since the works of H. Mori, R. Zwanzig, R. Picirelli, D. Zubarev, and many others, see [17-20]. In this approach, we obtain the hydrodynamic equations for macroparameters with some constitution equations relating to macrovariables. In general, such constitutive equations have the form of integrodifferential equations [15].

Let us make some comments concerning the reduced description. The reduction of a description takes place not only at the level of the BBGKY chain. Thus, even having the equations of hydrodynamics and solving them by approximate methods, we get the chain of reduced descriptions corresponding to different accuracies of methods. This is easy to see in the Galerkin method for the Navier-Stokes equations [21]. It is known that the construction of solutions in the form of a series in trigonometric functions leads to the system of three ordinary differential equations (the Lorentz system) with chaotic 
behavior. If we take a larger number of modes $M>3$ into account, then other types of chaotic or even periodic behavior are observed at $M>M^{\prime \prime}$.

It is clear that the different Galerkin systems derived with different numbers $M$ correspond to the different levels of reduced description (with $M$ leading parameters). This can help to understand the sense of such chaotic behavior and to answer the question: can the low-dimensional dynamical systems transfer the chaotic behavior of the initial system of PDEs.

Here, we can reason in the same way as in the case of the Liouville equations. If we had the complete phase portrait of the infinite-dimensional Galerkin system of ordinary differential equations (ODEs), we would pass the properties of a system of PDEs completely and would know the trajectories exactly.

In order to substantiate the low-dimensional dynamical system and the limiting transition as $M \rightarrow \infty$, there is a way appearing from the classical theory of difference schemes when the convergence and the accuracy are proven as $M \rightarrow \infty$.

However, presumably for any numerical scheme (at fixed $M$ ), a system with such a complicated phase portrait can be found that a numerical scheme will not be able to correctly pass the behavior of a solution. However, we do not need such detailed information about the solutions in many cases, since it is important to know only some general patterns. Here, the situation is the same as in the description of $N$ particles in a deterministic way or by means of the distribution function.

Therefore, the applicability of finite-dimensional dynamical systems as $M \rightarrow \infty$ should be considered in the sense of characteristics of chaos by the bringing of probabilistic conceptions (for example, the limit of invariant measures). In addition, it is well known that the application of probabilistic methods to the theory of cellular automata makes the proof easier [22].

Probabilistic considerations also allow to put forward a question about the correct transmission of chaotic behavior by means of different finite-dimensional projections. Some similar thing is done for the infinitedimensional Bogolyubov's chains in the so-called lattice systems [23].

We should especially note the reasons for the appearance of memory and the nature of memory in the given context. From Maxwell's considerations of the model example, the memory appears because of the accounting of a delay. In the thermodynamics of media with memory, the memory is simply postulated in integrodifferential relations for the flow of heat and the tensor of stresses with the defined kernels of integral relations.
But the exact type of relations and the specific expressions for kernels can be derived in statistical physics by means of a reduced description, when information about the "unimportant" degrees of freedom is taken into account implicitly through kernels. The essence of this phenomenon was found out in the so-called projection method of Mori-Zwanzig's type [24], and in the method of nonequilibrium statistical operator by D. Zubarev $[25,26]$.

In this approach, the distribution function $f$ is divided into two parts: $f=f_{1}+f_{2}$. For example, as a rule, $f_{1}$ is chosen as the locally Maxwellian distribution depending only on the relatively slowly changing hydrodynamic parameters $T, p$, and $u$. Part $f_{2}$ carries information about quickly changing micromotions. Then, the application of $f_{1}$ leads to hydrodynamic equations, which should contain contributions of the "rapid" part $f_{2}$.

In order to do this, the projection operator $P$ is introduced so that $f_{1}=P f, f_{2}=(1-P) f$. Then, one can use the solution of the Liouville equation in the form $f(t)=\exp (-i t L) f(0)$, where $L$ is the Liouville operator connected with the Hamiltonian system. After some manipulations [24, 26, 27], the expression for $f_{2}$ is obtained as

$f_{2}=e^{-i\left(t-t_{0}\right)(1-P)} f_{2}\left(t_{0}\right)-$
$-i \int_{0}^{t-t_{0}} d t^{\prime} e^{-i t^{\prime}(1-P) L}(1-P) L f_{1}\left(t-t^{\prime}\right)$.

The equation for $f_{1}$ is as follows:

$\frac{\partial f_{1}(t)}{\partial t}=-i P L f_{1}(t)-i P L e^{-i\left(t-t_{0}\right)(1-P)} f_{2}\left(t_{0}\right)-$

$-\int_{0}^{t-t_{0}} d t^{\prime} e^{-i t^{\prime}(1-P) L}(1-P) L f_{1}\left(t-t^{\prime}\right)$.

It easy to see that (2) and (3) have a temporal nonlocal character. This is caused by the division of the distribution function into two pieces. The difficulties with the choice of the projection operator are well known. They were overcome within the method of nonequilibrium statistical Zubarev's operator. According to this method, the distribution function depends on a certain collection of parameters of the reduced description $a_{1}, \ldots, a_{k}$, $f=f\left(a_{1}, \ldots, a_{k}\right)$, where $\left\{a_{j}\right\}$ are certain averages of the distribution function $f$ [26]. Note that, in this case, we can obtain, in principle, the same relations for $f$ taking the memory effect into account. 
Concerning the hydrodynamic level of description, the distribution functions lead to the equations of hydrodynamics in the form of balance laws for the momentum and the energy,

$\frac{\partial \rho}{\partial t}+\frac{\partial \rho V}{\partial x}=0$

$\frac{\partial V}{\partial t}+V_{k} \frac{\partial V_{k}}{\partial x}+\operatorname{grad} p=\operatorname{div} \sigma+F, \frac{\partial E}{\partial t}+\frac{\partial I_{E}}{\partial x}=0$

where $\rho$ is the density, $V$ is the velocity, $p$ is the pressure, $\sigma$ is the stress, $F$ is the thermal force, $E$ is the energy, $I_{E}$ is the energy flow.

To close these equations, the integral relations for the heat flow and the tensor of stresses are used. A short clear presentation of these issues is given in the appendix to the book written by Day [28].

Let us write these relations in the one-dimensional case:

$$
\begin{aligned}
& \sigma(x, t)=\int_{-\infty}^{t} d t^{\prime} \int d x^{\prime} K_{1} \frac{\partial u}{\partial x}\left(x^{\prime}, t^{\prime}\right), q(x, t)= \\
& =\int_{-\infty}^{t} d t^{\prime} \int d x^{\prime} K_{2} \frac{\partial T}{\partial x}\left(x^{\prime}, t^{\prime}\right) .
\end{aligned}
$$

In these relations, the kernels $K_{1}$ and $K_{2}$ derived by means of a routine procedure are connected with the correlators of variables. The correlators can be calculated with the help of the distribution function. For example, for $K_{1}$, we have

$$
K_{1}=\left(q(x), q_{1}\left(x^{\prime},-t\right)\right)
$$

Here, in (5), there is the ensemble average.

Thus, summing up the known results in statistical physics, it appears that the application of a reduced description at a hydrodynamic level leads to the conservation laws, which are closed by some integral relations. These integral equations can have a very complicated form including a nonlinear dependence on hydrodynamic variables. The specific type of kernels depends on a set of degrees of freedom involved in the process (this indicates the necessity of their incorporation at the reduced description).

In turn, these "necessary" degrees of freedom appear in a deviation from the equilibrium state. In equilibrium, thermodynamics is enough to characterize the temperature of a body as whole. It is easy to see that, without consideration of prehistory effects, the ordinary relations for the heat conductivity in the form of the Fourier law $q=-\lambda \partial T / \partial x$ and the relations for stresses $\sigma=-\nu \partial u / \partial x$ are derived. These relations lead to the parabolic equation of heat conduction and to the NavierStokes equations, respectively. The exponential kernels like $\exp (-t / \tau)$ lead to the hyperbolic equation of heat conduction and to the equations of relaxation hydrodynamics.

The exponential kernels correspond to a more exact consideration of a deviation from the equilibrium state. For an adequate, but rough description of the processes running far from equilibrium, the hydrodynamic equations may be enough at the beginning (it is possible with nonlinear constitutive relations). But after that, a hydrodynamic level can be not valid, and we need the whole one-particle distribution function $f_{1}$ (or even multiparticle distribution functions) as a parameter of the reduced description (see [29]).

Now, let us apply the aforesaid to the problem of turbulence. Assume that each level of reduced description has the corresponding characteristic chaotic behavior, which can be called the turbulence corresponding to the given level of reduced description. This was done in [30] in an implicit form and in [32] in an explicit form. A similar concept is contained in [31]. This assertion seems to allow us to reconcile the seemingly irreconcilable points of view on the so-called hydrodynamic turbulence. Of course, it is a complex phenomenon which can demand the complete description with the help of the function $f_{N}$ (for example, for the developed isotropic turbulence). However, the levels of reduced description may be enough for weaker turbulence and, especially, for the study of the initial stages of its development. Note that, at different levels of description, the features of a general picture may be different. That is why, despite the disadvantages of the Navier-Stokes equations, these equations can contain, nevertheless, information about the turbulent behavior. For example, in [4], the term "Navier-Stokes turbulence" appeared even. It is possible that the Navier-Stokes equations can pass the initial stages with the loss of the stability of laminar flows.

We observe other types of turbulent behavior, by using the levels of reduced description with the help of the complete Liouville equation. Thus, the place of many works concerning the incorporation of different fluctuations is cleared up. Small-scale fluctuations can be incorporated at the level of the Boltzmann equation in the integral term $([31,33]$ and others). Incorporation of large-scale fluctuations, which correspond to the collective motions like a fluid flow, is much more difficult. 
In this case, the Reynolds procedure of the separation into the average flow and pulsation components does not help, because it is based on the system of Navier-Stokes equations. Therefore, since the 1960s, plenty of work was devoted to the considerations of large-scale fluctuations. These works contain those rejecting the hypothesis of molecular chaos [34], the role of the asymmetry of distribution functions with respect to the transpositions of particles [35], etc.

So, in [36], the role of large-scale fluctuations had been accounted in the consideration of the equations for twoparticle distribution functions. The role of fluctuations was considered in [31] as well. It is worth noting book [37], which is not devoted especially to the problem of turbulence, but contains a lot of results having a direct relation to it.

Thus, in principle, it is known how to construct the equations corresponding to the levels of reduced description in a general abstract form. As a rule, theoretical physics deals with the qualitative investigation of these equations. However, the specific information extracted from them is difficult for the interpretation because of their complication. At the same time, in hydrodynamics, it would be desirable to have the description of macroeffects. In this case, the study of model equations can help. Within this approach, the Navier-Stokes equations and the equations for Maxwell's media can be regarded as model equations. As mentioned above, the usefulness of model equations for the consideration of turbulence is confirmed by both experimental data on the turbulent flows and their derivation from the Boltzmann equation. Note that they can be obtained with the use of the model exponential kernels given by relations (4).

It is possible also to assume that the same relations with exponential kernels will be a model in some sense for equations with an extended set of variables in comparison with the hydrodynamic one, describing the average flows. The characteristic times of relaxation determined by large-scale fluctuations can be large enough in comparison with the free motion time of molecules, as experiments demonstrate. Moreover, it is possible to assume that the equations of hydrodynamics with memory are, in some sense, the case of "general position" at the modeling of turbulence, while the Navier-Stokes system is a degenerate case where the time of relaxation tends to zero.

So, it is possible to make conclusion that, at the modeling of turbulence, the equations with memory are more preferable. Therefore, in order to make some features of turbulence on the hydrodynamic stage more exact, we should use the presented equations or their consequences.

It turns out that the consideration of equations with memory or their model equations leads to some unexpected results and raise problems, some of which are given in the following sections.

We also note that some reduction procedures can be applied further to hydrodynamic equations. For example, the search for a small number of leading parameters in the theory of dissipative structures or synergetics and in the phase-transition theory can be also considered as a reduction in description (see the works by I. Prigogine, H. Haken, W. Ebeling). We also mention the new investigations of reducing the partial differential equations to low-dimensional ordinary differential equations and their attractors $[38,39]$. The next stage of reduction consists of the transition to the pure thermodynamic description. It should be stressed that, in general, the precise abstract equations for different hierarchical levels of description are known from theoretical physics. Usually, theoretical physicists explore these very complex abstract equations (frequently qualitatively). But, on a hydrodynamic level, it is especially interesting to search for visible macroeffects. In such a case, the consideration of model equations is especially useful. Thus, the Navier-Stokes equations, their hyperbolic counterpart, and low-dimensional systems of ordinary differential equations are the model equations.

There is a great interest in the investigation of typical model equations for different hierarchical levels and their typical solutions. Note that, usually, such typical solutions are the basic elementary objects for a description of complex real phenomena.

\section{Collapses, Elementary Objects, and Instabilities}

As was mentioned in the previous sections, various formations play an important role in turbulence. To some degree, the type of structures under consideration determines possible functional spaces. Therefore, the results presented here largely join the results of the previous section.

Among elementary objects, we should first distinguish the singularities of solutions. In other words, these are collapses, modes with intensification, and blow-up solutions. These solutions tend to infinity for a finite time at certain points. Despite the exoticism, such solutions are often used in the investigations of physical processes. It turns out that they have a long history connected with problems of turbulence. For example, the vortical tubes 
in a fluid at the pull increase the rotation. The dissipation of energy in turbulence takes place not in the whole fluid, but it is concentrated in the certain localized regions, which is known as the intermittency. Vortices can grow under conditions of rotation of turbulent fluids.

Naturally, the phenomena of such types are represented in the mathematical models of turbulence. One class of effects is connected with the vortical motions of ideal incompressible fluid which is a good model for the study of the developed turbulence, when the Reynolds number $\operatorname{Re} \rightarrow 0$, and the influence of viscosity is ignored. Except for pure experimental works, there is a large number of investigations on the numerical modeling. According to the results of modeling, it has been revealed that, under the conditions of precise computations with the help of special numerical schemes, there is a tendency to the increase of vorticity with regard for the character of approximating methods (see [40]). Finally, the exact analytic solutions with a vorticity collapse are found.

Singularities of the initial Navier-Stokes system are a much more difficult problem. Let us recall the block of ideas related to the theory of turbulence such as the supposition that a solution or its functionals is referred only to a finite moment of time and then tends to infinity. After the moment of collapse, the solution "becomes" finite again; then the process is repeated. Note that nonuniqueness can be observed. Therefore, we should keep in mind the classical investigations concerning the theorems of existence and uniqueness of solutions of the Navier-Stokes equations. We note that J. Leray proved the existence of a global weak solution in the class of quadratically summable functions in the three-dimensional case under the condition that the initial data have bounded energy.

However, the existence of such solutions does not guarantee the absence of a singularity of the solution (i.e., the increase of a solution to infinity). It was also unknown, whether such solutions can develop from smooth initial data. The essential results in this direction are the derivation of a strong upper bound for the Hausdorff dimension. It turns out that the measure of the support of singularities is equal to zero.

Recently, this problem has been developed in papers related to the multi-valued solutions. As a consequence, the concept of "concentration in the solution" appeared. This means that a sequence poorly converging in $L_{2}$ functions can have a limiting function with the infinite values of solutions on the "emaciated" set. As an example, the sequence of functions in $R^{n}$ [41] is presented. The work of F. Merle is indicated in [41], where the behavior of a solution after the infinity moment is considered.

Let us pay attention to work [42] which deals with singular solutions. There, for the Burgers equations and the boundary-value problem, the classes of stationary solutions having singularities were studied.

Perhaps, the first solution of the Navier-Stokes system with a collapse was considered in [43]. In connection with a collapse, let us point out that the studies were carried out for model equations of the hyperbolic type of the second order.

The collapse in a hydrodynamic system with internal degrees of freedom was considered numerically by V.A. Khrisheniuk. These results are based on strict mathematical assertions. In spite of many open questions, the solutions with a singularity of the vortical type were repeatedly used in different physical investigations. So, in [44], the dynamical system consisting of a small number of vortices is considered, and the conditions for the appearance of chaotic motions are stated. The subsequent investigations were developed toward a higher number of vortices, their grids, and the distribution of vortices in media, when the statistics of objects-vortices was built. We also indicate the chaos built with regard for the co-operation of vortices and spiral waves.

In connection with the hydro- and gas-dynamics, we mention another type of singularities related to the socalled gradient catastrophes, when the solutions remain bounded, but the spatial derivatives grow without any restriction. Thus, there can be shock waves in the solution. Such a situation is especially characteristic of the compressible media. Moreover, such shock waves or solutions formed from their combinations serve the elementary solutions in some models of turbulence, when a model is built on the statistics of such excitations (for example, turbulence in the Burgers model).

V.E. Zakharov and co-workers dealt with collapses considering them both numerically and analytically [45, 46]. The brief, but very capacious review is presented in [47], where the possible applications of collapses to hydrodynamic turbulence are announced. The role of collapses is especially interesting for plasma as places of dissipation (flow of energy), as well as their role in Langmuir turbulence, when high-frequency vibrations are concluded in a small-size cavity. These phenomena were predicted in a theory and confirmed by computations. Moreover, they found experimental confirmation quite recently [48]. It is important that this process can go in the retrograde direction on the spectrum of waves, i.e. the energy is passed into a short-wave region. This differs from many models, in which the energy is passed 
over a spectrum into a long-wave region, where the condensation of long-wave excitations can become even similar to the Bose-condensation in quantum theory. In [47], the types of collapses, the amount of the energy involved, and the threshold effects were considered. There, the basic model equation (as well as in many other works) is a nonlinear Schrödinger equation. Because of the importance of the hypotheses mentioned in [47], we will quote two extracts:

"The multiple development of wave collapses, where the dissipation of turbulent energy occurs, is characteristic of many types of turbulence in a continuous medium. It is not set presently, whether the classical turbulence of ideal incompressible fluid belongs to the given type, although there are very serious grounds for this hypothesis ..." "However, plasma physics is the basic "user" of theory of wave collapses."

As our investigations have shown, the more general models of hydrodynamics considering the effects of memory give, indeed, serious arguments in favor of such a hypothesis. Note that the role of singularities in turbulence was described in [30]. Dispersion in the system is also important. In connection with collapses, the solitoninvolved turbulence was also considered, when the modulation instability is a reason for the appearance of solitons as elementary objects making turbulence.

Work [49], in which the collapses are considered in a conceptual plan from a new point of view, recently appeared (this paper intersects partially with the cited articles). It also deals with solutions with the development of collapses on the basis of the nonlinear Schrödinger equation used adequately describe the abnormally large transfer in turbulent flows. The model is built in such a way that the solutions with collapses do not always grow, and, at large amplitudes, some limiting factors result in a reduction of the amplitudes. This process repeats itself by the spontaneous appearance and is named the homoclinic excursion. It looks like that a model passes, indeed, some lines to turbulence. However, the authors of [49] discussed the question about the applicability of the Schrödinger equation to the real hydrodynamic turbulence. Let us note that the equations involving the memory allow one to consider similar effects.

The numerous investigations described in the practically similar books on turbulence and chaos indicate that the structures usually arise as a result of the display of different nonstabilities in the systems. The type of an instability determines the character, size, and dynamics of structures. Therefore, concluding this section, we will briefly discuss different types of instability, because some definite advancements appeared lately in this di- rection. In addition, as is generally known from works on dynamic chaos, it is considered that a local instability provides this phenomenon.

There is an enormous number of investigations on hydrodynamic instabilities. Here, we refer only to a small number of topics. The first topic concerns the problems of incorrectness in physical problems. It is known that the physical problems should be posed so that they are correct by Hadamard. At the same time, many classes of ill-posed problems exist. In hydrodynamics, such problems arise as well. We recall, for example, the problems of the explosive instability with the Burgers equations with the negative coefficient of viscosity, and the problems of the boundaries of flows (the Kelvin-Helmgoltz instability). A noncorrectness arises in the problems of motion in viscoelastic media under the change of their type from hyperbolic to elliptic. The Cauchy problems for elliptic equations arise in investigations of elliptic equations for defects. Recently, some ill-posed problems were considered by V.P. Maslov [50]. He gave the definitions of degree of noncorrectness [51].

In hydrodynamics, V.P. Maslov considered the asymptotic behavior of viscosity $\nu \rightarrow 0$ with fast-oscillating initial data, which leads to the asymptotic uniqueness and the loss of causality in deterministic problems [50]. With collapses, some unsteady problems were considered, when a solution grows due to resonances.

Here, we briefly describe the phenomenon of waves with negative energy in media with memory. Such waves were first considered in the problems of electronics in the 1950s. The essence of the phenomenon consists in definite wave disturbances of the distributed active medium such that the growth of their amplitudes is accompanied by a decrease of the energy of the media-wave system [52]. Formally, it is expressed in the negative distribution of energy for these waves. Essentially, such effects are possible only in thermodynamically nonequilibrium media. It is also known that the frequent co-operation of waves with negative energy results in collapses, and the properties of such systems are determined by the dispersion relations of linearized problems.

In conclusion, we will indicate another aspect related to the instability, turbulence, and chaos. As was already mentioned, for the chaos (in the mathematical sense) in finite-dimensional systems, a instability (the Oseledets theorem) is needed. However, this situation is not simple for the infinite-dimensional systems.

In addition, there is a separate complex problem related to the supervision of chaos in the infinitedimensional systems and their interpretation. In fact, it is similar with the problem of image regeneration, 
which, as is generally known, is improper. There are many examples of effects of this type, and these problems deserve a deeper study. We mention the intricate mathematical problems of stability in systems with singular coefficients (as those described by the Schrödinger equation with singular potential).

\section{Low-Dimensional Models for Two-Dimensional Generalized Hydrodynamics}

Among approaches to the investigation of hydrodynamic equations, the Galerkin method should be distinguished. Using this method, it is easy to calculate a low-dimensional dynamical system. So, we will deal with dynamical systems obtained from the nonlocal hydrodynamic models presented above.

Since the generalized models can be regarded as singular perturbations of simpler models, it is useful to carry out the comparison of the solutions for dynamical systems squeezed out from various models of hydrodynamics.

In particular, such test-systems are the well-known Lorentz system and the low-dimensional system for plane flows investigated in [53].

\subsection{Finite-dimensional systems for two-dimensional flows with the periodicity condition}

One of the models considering the nonlocality effects in two spatial dimensions is the Oldroyd fluid (the generalization of the Maxwell medium ([12-14]):

$$
\begin{aligned}
& \frac{\partial \bar{V}}{\partial t}+V_{k} \frac{\partial \bar{V}}{\partial x_{k}}+\tau\left(\frac{\partial^{2} \bar{V}}{\partial t^{2}}+\frac{\partial V_{k}}{\partial t} \frac{\partial \bar{V}}{\partial x_{k}}+V_{k} \frac{\partial^{2} \bar{V}}{\partial t \partial x_{k}}\right)_{k}- \\
& -\nu \Delta \bar{V}-\mu \frac{\partial \Delta \bar{V}}{\partial t}=-\left(1+\tau \frac{\partial}{\partial t}\right) \operatorname{grad} P+\bar{F}(x, t)
\end{aligned}
$$

$\operatorname{div} \bar{V}=0$

where $\bar{V}=\left\{V_{1}, V_{2}\right\}$ are velocity components, $\nu$ is the viscosity coefficient, $P$ is the pressure, $\bar{F}=\left\{\bar{F}_{1}, \bar{F}_{2}\right\}$ are external forces, $\tau$ is the relaxation time, $\mu$ is the nonlocality coefficient, and $(\cdot)_{k}$ means the summation over repeated indices.

We consider model (6) on a plane region $T^{2}=[0,2 \pi] \times$ $[0,2 \pi]$ with periodic boundary conditions. The geometry of a flow and the boundary conditions were imposed just like in [53]. In such a case, we consider the flows with the velocity component $v_{z}=0$. We assume that the flow is space-periodic in the $(x, y)$-plane with period $2 \pi$. This implies that the flow and the flow derivatives are periodic with period $2 \pi$. Moreover, we also assume the mean flow averages over the region $T^{2}$ (see [53] and [38]):

$\int_{T^{2}} \hat{V} d x=0$

In our case, the solution is considered as a series in harmonics $\exp (i \hat{k} \hat{x})$, where $\hat{x}$ are coordinates, and $\hat{k}$ are wave vectors with integer components,

$\hat{v}(x, t)=\sum_{k=1}^{\infty} \gamma_{k}(t) \exp (i k x)$,

where $k$ is the wave vector.

Inserting (7) into (6) and performing long calculations, we obtained the system of equations for coefficients $\gamma_{k}(t)$ :

$\tau \frac{\partial^{2} \gamma_{k}}{\partial t^{2}}+\frac{\partial \gamma_{k}}{\partial t}=$

$=-i \sum_{k_{1}+k_{2}+k} \gamma_{k_{1}} \gamma_{k_{2}}\left(k_{1}+k_{2}\right)\left[1-\frac{k_{1}\left(k_{1}+k_{2}\right)}{|k|^{2}}\right]-$

$-\nu\left|k^{2}\right| \gamma_{k}+f_{1}(k) \frac{k_{2}^{2}}{|k|^{2}}-$

$-i \tau\left[\sum_{k_{1}+k_{2}+k}\left(\gamma_{k_{1}} \frac{\partial \gamma_{k_{2}}}{\partial t} \gamma_{k_{2}} \frac{\partial \gamma_{k_{1}}}{\partial t}\right)\left(k_{1}+k_{2}\right)\left(1-k_{1}-k_{2}\right)\right]+$

$+\tau \frac{\partial f_{1}}{\partial t}\left(1-i \frac{k_{1}}{k^{2}}\right)-i \tau \frac{k_{1}}{k^{2}} \frac{\partial f_{2}}{\partial t}$,

where $f_{1}$ and $f_{2}$ correspond to the Fourier expansion of the function $F$. This infinite-dimensional system is equivalent to the original system of PDEs. However, its investigation is a very difficult problem.

Therefore, it is of common practice to "cut-off" such a system and to merely consider a finite number of its components. Truncated systems usually give some information about the behavior of original equations. Thus, we must take $k$ in (7) from some bounded set $L$. Let us take $L=\left\{k_{1}=(1,1), k_{2}=(3,0), k_{3}=(2,-1), k_{4}=\right.$ 
$\left.(1,2), k_{5}=(0,1)\right\}$ plus the vectors opposite in sign. After the reduction, the following low-dimensional system was obtained $(\nu=1.0)$ [53]:

$$
\begin{aligned}
& \dot{\gamma}_{1}=2 \gamma_{1}+4 \gamma_{2} \gamma_{3}+4 \gamma_{4} \gamma_{5}, \quad \dot{\gamma}_{2}=-9 \gamma_{2}+3 \gamma_{1} \gamma_{3} \\
& \dot{\gamma}_{3}=-5 \gamma_{3}-7 \gamma_{1} \gamma_{2}+r \\
& \dot{\gamma}_{4}=-5 \gamma_{4}-\gamma_{1} \gamma_{5}, \quad \dot{\gamma}_{5}=-\gamma_{5}-3 \gamma_{1} \gamma_{4} .
\end{aligned}
$$

The memory effects lead to the ten-dimensional counterpart of $(9)$ :

$$
\begin{aligned}
& \frac{d x_{1}}{d t}=\frac{-x_{1}-2 x_{6}+4 x_{7} x_{8}+4 x_{9} x_{10}}{\tau}+ \\
& +4\left(x_{2} x_{8}+x_{7} x_{3}\right)+4\left(x_{4} x_{10}+x_{9} x_{5}\right)-2 \mu x_{1}, \\
& \frac{d x_{2}}{d t}=\frac{-x_{2}-9 x_{7}+3 x_{6} x_{8}}{\tau}+3\left(x_{1} x_{8}+x_{6} x_{3}\right)-9 \mu x_{2}, \\
& \frac{d x_{3}}{d t}=\frac{-x_{3}-5 x_{8}-7 x_{6} x_{7}}{\tau}- \\
& -7\left(x_{1} x_{7}+x_{6} x_{2}\right)-5 \mu x_{3}+\frac{R}{\tau}, \\
& \frac{d x_{4}}{d t}=\frac{-x_{4}-5 x_{9}-x_{6} x_{10}}{\tau}-\left(x_{1} x_{10}+x_{6} x_{5}\right)-5 \mu x_{1}, \\
& \frac{d x_{5}}{d t}=\frac{-x_{5}-x_{10}-3 x_{6} x_{9}}{\tau}-3\left(x_{1} x_{9}+x_{6} x_{4}\right)-\mu x_{5}, \\
& \frac{d x_{6}}{d t}=x_{1}, \quad \frac{d x_{7}}{d t}=x_{2}, \quad \frac{d t}{d t}=x_{3},
\end{aligned}
$$$$
\frac{d x_{9}}{d t}=x_{4}, \quad \frac{d x_{10}}{d t}=x_{5}
$$

Now, we are going to describe some properties of system (10). It is easily seen that the stationary points for the original variables $\left(x_{6}, \ldots, x_{10}\right)$ coincide with the stationary points for 5 -dimensional systems from [53] (because $x_{i} \equiv 0, i=1, \ldots, 5$ must be fulfilled in steady states for (10)). This is because the complement terms in (6) with coefficients $\tau, \mu$ have time derivatives. Thus, the difference in stationary points between the usual and generalized cases consists in stability properties. The full investigation of ODE systems for generalized hydrodynamics is a forthcoming problem (especially in cases of high-dimensional systems), so now we present only some numerical results.

We carried out the numerical calculations of the dynamical system (10), which demonstrate a complicated new irregular behavior of the solution. This type of a phase portrait (called the butterfly) corresponds to the Lorentz-type chaos. The behavior becomes much more complicated, as the memory is considered. It is characterized by a large value of maximal Lyapunov exponent (which is larger than 1) and a broad power spectrum. The projections of trajectories have a cross-like shape. Let us note that such forms were found earlier in the seven-dimensional system for the Navier-Stokes equations [54]. In analogy with [54], we may suppose that such a pattern is created when the eigenvalue of the Poincaré map for the periodic orbits crosses the unit circle at the point +1 , and when the nonsteady hyperbolictype orbits are created.

According to the numerical integration, if $R$ is increased, the amplitude of the limit cycle grows until the stability is lost, because the double bifurcation of the period occurs. The subsequent development of the periodic regime was studied with the help of the Poincaré bifurcation diagram. We can distinguish several perioddoubling bifurcations attached to the chaotic region in the diagram. It is possible to make conclusion from the form of the chaotic region that two different types of chaotic attractors interact in the phase space of the dynamical system (10).

Thus, it follows from the obtained results that the memory effects can cause a more complicated behavior of solutions of the hydrodynamic model, while the nonlocality can play the stabilizing role (at least in the considered cases).

\section{Model for a Three-Dimensional Case}

Low-dimensional dynamical systems were constructed for three-dimensional flows. Among results for such a system, it is necessary to point out two new features. First, the flow with external force demonstrates the intermittency-II-type behavior under certain conditions. Second, autooscillations were observed even without external forces.

Continuing the investigations of model (6), let us take the boundary conditions for the three-dimensional system (6) in the form

$$
\left.\bar{V}\right|_{\partial \Omega}=0
$$


where $\Omega \in R^{3}$ is a region bounded by the surface $\partial \Omega$, which is the rigid boundary of the fluid volume. Relation (11) known as the "stick" boundary condition means that the velocity $\mathbf{V}$ vanishes on the rigid boundary (nonslip boundary conditions). Physically, this means that fluid does not move near the boundary, $\mathbf{V}_{\partial \Omega}=0$.

According to the Galerkin method, the solutions of model (6) are looked for as the expansions

$V(x, t)=\sum_{k} z_{k}(t) \Phi_{k}(x)$,

where $\left\{\Phi_{k}(x)\right\}$ form the full system of orthogonal eigenfunctions for the linear eigenvalue problem with eigenvalues $\mu_{k}$ and eigenfunctions $\Phi_{k}$ :

$\nu \Delta \Phi_{k}=-\mu_{k} \Phi_{k}+\operatorname{grad} p_{k}, \quad \operatorname{div} \Phi_{k}=0$,

$\left.\Phi_{k}\right|_{\partial \Omega}=0, \quad \int_{\Omega} \Phi_{k}(x) d x=1$.

After some lengthy computations, we get the system for amplitudes $z_{k}, k \in N$

$$
\begin{aligned}
& \tau \frac{d^{2} z_{\ell}}{d t^{2}}+\frac{d z_{\ell}}{d t}+\sum_{k} \sum_{m} c_{k m \ell} z_{k} z_{m}+\mu_{\ell} z_{\ell}+ \\
& +\tau \sum_{k} \sum_{m}\left\{z_{m} \frac{d z_{k}}{d t}+z_{k} \frac{d z_{m}}{d t}+\nu \frac{d z_{\ell}}{d t}\right\} c_{k m \ell}=f^{\ell},
\end{aligned}
$$

where $\mu_{\ell}, \Phi_{\ell}$ are given by (13),

$f^{\ell}=\int_{\Omega} \bar{F} \Phi^{\ell} d x, c_{k m \ell}=\int_{\Omega}(\Phi, \nabla) \Phi_{m} \Phi_{k} d x, \ell \in N$.

The values of $f^{\ell}$ and $c_{k m \ell}$ coincide with the analogous values from [55]. It is seen that, for $\tau=\mu=0$, system (14) coincides with that obtained in $[39,55]$ for the Navier-Stokes equations. To investigate the influence of memory and nonlocality on truncated Galerkin approximations, we take the system of ODEs from [55]:

$$
\begin{aligned}
& \frac{d x_{1}}{d t}=-\eta_{1} x_{1}+A x_{2} x_{3}+F_{1}, \\
& \frac{d x_{2}}{d t}=-\eta_{2} x_{2}+B x_{1} x_{3}+F_{2}, \\
& \frac{d x_{3}}{d t}=-\eta_{3} x_{3}+C x_{1} x_{2}+F_{3} .
\end{aligned}
$$

Here, $A, B, C$, and $F_{i}$ are constants, and the additional relation $A+B+C=0$ is satisfied. The last condition is derived in [55] by from considering the kinetic energy of a nonviscous fluid with nullifying mass forces $(\nu=0$ $, \bar{F}=0)$. Let us fix the parameters

$A=1, B=-2, C=1$

$F_{1}=-\frac{-5+\sqrt{76}}{2}, F_{2}=-20-\sqrt{76}, F_{3}=\frac{34+5 \sqrt{76}}{4}$,

$\eta_{i}=\nu, \quad i=1,2,3$

for illustration. Then the low-dimensional system in this case is

$\frac{d x_{1}}{d t}=\frac{-x_{1}-x_{5} x_{6}-\nu x_{4}+F_{1}}{\tau}-\left(x_{3} x_{5}+x_{6} x_{2}\right)-$

$-\nu \operatorname{sgn}|\mu| \mathrm{x}_{1}$,

$\frac{d x_{2}}{d t}=\frac{-x_{2}+2 x_{4} x_{6}-\nu x_{5}+F_{2}}{\tau}+2\left(x_{1} x_{6}+x_{3} x_{4}\right)+$

$+\nu \operatorname{sgn}|\mu| \mathrm{x}_{2}$,

$\frac{d x_{3}}{d t}=\frac{-x_{3}-x_{4} x_{5}-\nu x_{6}+F_{3}}{\tau}-\left(x_{2} x_{3}+x_{1} x_{5}\right)-$

$-\nu \operatorname{sgn}|\mu| \mathrm{x}_{3}$,

$\frac{d x_{4}}{d t}=x_{1}, \frac{d x_{5}}{d t}=x_{2}, \frac{d x_{6}}{d t}=x_{3}$

It was shown in [55] that, in the case of the NavierStokes system, there is a Hopf bifurcation, when $\nu=0$. The value $\nu_{c r}=2$ separates the steady state and the region with periodic orbits. There is only a stable equilibrium for $\bar{F}=0$. Now, let us present the results of numerical integration of system (18) performed with the help of the Runge-Kutta method. We observe the growth of amplitudes, decaying oscillations, and the almost constant solution on a time interval of about five time units. However, near a time of 9.0, there is a strong burst of the solution. After a time of 12.0, the burst decays. Then, after a time of 14.0, the burst takes place again. This is similar with the type-II intermittency.

Another interesting result is an oscillating regime without mass forces. These oscillations take place for 
nonzero initial values. This case can be interpreted as the evolution of a flow without external influence. The presented behavior illustrates one of the possible ways of the explanation of turbulence in flows without restoring to negative viscosity. Our computations also confirm the stabilizing role of nonlocality.

Let us summarize the numerical and analytical results of the investigations of systems (16), (18), (9), and (10).

For the six-dimensional system (18), we found the appearance of periodic solutions. Such solutions were also found without external forces $(F=0)$ under nonzero initial conditions. We also found, in some cases, the phenomena similar to "intermittency" (bursts in solutions). The ten-dimensional system (10) was investigated for some parameters. We changed the values of relaxation time and the initial conditions. In the case with no memory $(\tau=0)$, the projections of the phase portrait on two-dimensional planes have the "butterfly" type of an attractor similar to the diagrams for the Lorentz chaotic attractor. In the case with $\tau \neq 0$, there is a complex behavior of a new type. Visually, the trajectory fills densely a bounded volume (named "container"). Trajectory projections on the planes have a broken form in many points. Locally, the projection of the phase portrait looks like a ball of thread or "patience". Visually, the behavior is similar to two-dimensional mappings with homoclinic tangency and quasiattractors, which was described in [56]. Similar pictures were found in systems with the so-called "fat-fractals" with larger fractal dimension than that of a Cantor-type set. In many cases, the results of numerical calculations look like the projections of motion on the torus. The first Lyapunov characteristic exponent stayed positive for a long time (but with diminishing value). The numerical investigation of (10) with different values of $R$ displayed the still excitation of a complex behavior. The standard one-dimensional bifurcation diagram is entirely different from the usual perioddoubling scenario of the transition to chaos. We also made some numerical investigations of the bifurcation for a 10-dimensional system. We found that, without external forces $(R=0)$, there was a unique stationary point with zero coordinates. The Jacobian of the right part of (10) had a pair of pure imaginary eigenvalues. Some further bifurcation was obtained by increasing the number of stationary points to ten with increasing the value of $R$. At this process, the pairs of complex conjugate eigenvalues cross the imaginary axis from left to right. Further investigations of the systems above look very promising in the case $\tau \ll 1$. This is the singular perturbation of the usual systems of ODEs with chaos. The evaluation of bounds for the attractor dimension in the case $\tau \rightarrow 0$ is of interest (especially in the limit $N$ or/and $R$ tending to infinity). Note that (14) consists of ODEs of the second order in time. Hence, such a system reminds the collection of oscillators. Each $z_{k}$ corresponds to a wave number of the harmonic $k$. So, we may anticipate the properties like the transmission of energy along the spectrum of harmonics, the existence of harmonics clusters, resonances, and so on. Also, some ideas of memory effects in turbulence may be reconsidered. There are many space and time scales in fluid flows. This yields the existence of many types of chaotic behavior in fluids. The account of memory leads presumably to a new type of chaos similar to the chaos in media constructed from oscillators. Let us also note that the above complex behavior may serve as a prototype of a new possible type of chaos in media with finite speed of propagation and with gauge symmetry.

\section{Applications of Model Equations to Physical Processes}

The proposed models are quite new and are useful objects for further mathematical investigations. But just now, some interpretation of the solutions may be proposed for real physical processes. Some applications to heat and mass transfer processes had been proposed and tested experimentally earlier: the hyperbolic heat conduction equation, ignition theory by heat explosion, and heat and mass transfer in turbulent and heterogeneous media (see [14]). New peculiarities of solutions found for new model equations allow the consideration of some aspects of very important recent physical and technical problems, where strongly nonequilibrium properties are essential. A wide range of applications is represented by the processes in astrophysics. The first subclass of problems is the description of the phenomena in the sparse plasma, including also MHD. The second relevant field of applications is the processes in the near-Earth space, where a great number of different structures have been found experimentally. The third field of investigations is the processes in the Sun, especially the formation of hot and cool spots, arcs, bursts, cellular lattices on the surface, etc. Many theories have been proposed earlier, but the problems are still open. The most interesting consequence of the proposed model equations for such problems is the presumable fragmentation of a flow with diminishing the scale and increasing the vorticity of the amplitudes of solutions for fast flows. Other important problem is the building of thermonuclear power plants. One of the main problems is the control over such processes and apparatus and the suppression of a wide spec- 
trum of possible instabilities. Usually, the classical MHD equations are used. But the proposed considerations lead to the conclusion that new more accurate equations for such processes far from equilibrium are needed. Finally, the proposed model equations with memory and nonlocality effects can be used for extending the classical synergetic problems to the processes much far from equilibrium in the usual synergetics. We note, for example, that the synergetics includes the classical KuramotoSivashinsky equation for considering many kinds of instabilities. So, for the processes far from equilibrium, the new equations to investigate the instabilities can be proposed. Such equations will be a generalization of the Kuramoto-Sivashinsky equation with regard for memory and nonlocality effects. For example, one of such equations (with one spatial variable) for the formation of structures has the form:

$\tau \frac{\partial^{2} u}{\partial t^{2}}+\frac{\partial u}{\partial t}+u \frac{\partial u}{\partial x}+\nu \frac{\partial^{2} u}{\partial x^{2}}+\alpha \frac{\partial^{3} u}{\partial x^{3}}+\beta \frac{\partial^{4} u}{\partial x^{4}}=0$,

where $\alpha, \beta, \mu, \tau$, and $\nu$ are parameters. Note that the ordinary differential equation with a solution of the "traveling wave" form coincides with that in the classical case $(\tau=0)$, but the stability conditions for such a solution are different from those in the classical case.

\section{Conclusions}

Thus, in the present work, we propose to extend the studies of memory effects in some distributed systems allowing the classical description by partial differential and ordinary differential equations. This leads to new mathematical problems concerning the study of these new objects and their solutions. The first studies allowed us to find new properties and to propose a lot of new research problems.

1. L.D. Landau and E.M. Lifshitz, Fluid Mechanics (Pergamon Press, Oxford, 1975).

2. A.S. Monin, P.Ya. Polubarinova-Kochina, and V.I. Khlebnikov, Cosmology, Hydrodynamics, Turbulence: A.A. Friedmann and the Development of His Scientific Heritage (Nauka, Moscow, 1989) (in Russian).

3. Yu.L. Klimontovich, Statistical Physics (Harwood, New York, 1986).

4. R.H. Kraichnan, in New Trends in Turbulence (Springer, Berlin, 1991), p. 1.

5. D. Ruelle, in New Trends in Turbulence (Springer, Berlin, 1991), p. 123.

6. M. Lesier, Turbulence in Fluids (Kluwer, Dordrecht, 1997).
7. B.B. Kadomtsev, Collective Phenomena in Plasmas (Pergamon Press, New York, 1982).

8. V.N. Tsytovich, Theory of Turbulent Plasma (Consultants Bureau, New York, 1977).

9. V.N. Tsytovich, Usp. Fiz. Nauk 108, 143 (1972).

10. V.D. Shapiro and V.I. Shevchenko, in Fundamentals of Plasma Physics (Energoatomizdat, Moscow, 1984), Vol. 2, p. 119 (in Russian).

11. G.K. Batchelor, An Introduction to Fluid Dynamics (Cambridge Univ. Press, London, 1967).

12. A.P. Oskolkov, Trudy Math. Inst. AN BSSR 159, 101 (1983).

13. A.P. Oskolkov, Trudy Mat. Inst. Steklov. 179, 126 (1988).

14. A.S. Makarenko and T. Swirsky, in Proc. of the $X V$-th Int. Hutsulian Workshop "Category theory and Applied System Analysis in Decision-Mking Problems" (Kyiv, 2006), p. 183.

15. V.A. Danylenko, T.B. Danevych, O.S. Makarenko, S.I. Skurativskyi, and V.A. Vladimirov, SelfOrganization in Nonlocal Nonequilibrium Media (Subbotin Inst. of Geophys., Kiev, 2011).

16. V.A. Danylenko, V.Yu. Korolevich, A.S. Makarenko, and V.O. Christenyuk, Self-Organization in Strongly Nonequilibrium Media. Collapses and Structures, Preprint (Subbotin Inst. of Geophys., Kiev, 1992).

17. P. Resibois and M. de Leener, Classical Kinetic Theory of Fluids (Wiley, New York, 1977).

18. D. Zubarev and S. Tishchenko, Physica 59, 285 (1972).

19. D.N. Zubarev and M.V. Tokarchuk, Teor. Mat. Fiz. 70, 234 (1987).

20. A.S. Makarenko, M. Moskalkov, and S. Levkov, Phys. Lett. A 23, 391 (1997).

21. R. Temam, The Navier-Stokes equation. Theory and Numerical Analysis (Amer. Math. Soc., Providence, RI, 2001).

22. E. Presutti, in Stochastic Processes. Mathematics and Physics II, edited by S. Albeverio, P. Blanchard, and L. Streit (Springer, Berlin, 1987), Vol. 1250, p. 195.

23. A.K. Vidybida, Teor. Mat. Fiz. 68, 69 (1986).

24. R. Zwanzig, J. Chem. Phys. 33, 1338 (1960).

25. D.N. Zubarev, Nonequilibrium Statistical Thermodynamics (Consultants Bureau, New York, 1974)

26. D.N. Zubarev and A.M. Khazanov, Teor. Mat. Fiz. 34, 69 (1978).

27. R.A. Picirelli, Phys. Rev. 175, 77 (1968).

28. W.A. Day, The Thermodynamics of Simple Materials with Fading Memory (Springer, Berlin, 1972).

29. D.N. Zubarev, Teor. Mat. Fiz. 53, 93 (1982). 
30. V.A. Kuleshov and A.S. Makarenko, Depos. AISHCI, No. 7491 (AISHCI, Moscow, 1985) (in Russian); J. Engrg. Phys. 48, 664 (1985).

31. Yu.L. Klimontovich, Turbulent Motion and the Structures of Chaos (Kluwer, Dordrecht, 1991).

32. A.S. Makarenko, Depos. Ukr. Nat. Inst. Techn. Inf., No. 1504 (UNITI, Kiev, 1988) (in Russian).

33. R.L. Liboff, Introduction to the Theory of Kinetic Equations (Wiley, New York, 1969).

34. R.N. Zhigulov, in Turbulent Flows (Nauka, Moscow, 1974), p. 75 (in Russian).

35. T. Koga, Introduction to Kinetic Theory of Stochastic Processes in Gaseous Systems (Pergamon, Oxford, 1970).

36. A.D. Khonkin, in Turbulent Flows (Nauka, Moscow, 1974), p. 141 (in Russian).

37. O.A. Grechannyi, The Statistical Theory of Irreversible Processes (Naukova Dumka, Kiev, 1989) (in Russian).

38. R. Temam, Infinite-Dimensional Dynamical Systems in Mechanics and Physics (Springer, New York, 1988).

39. O.A. Ladyzhenskaya, Mathematical Questions of the Dynamics of a Viscous Incompressible Fluid (Moscow, Nauka, 1970) (in Russian).

40. R. Krasny, in Proc. of Intern. Congress of Math., Kyoto, Japan (Math. Soc. Japan, Tokyo, 1990), p. 1573.

41. A.J. Majda, in Proc. of Intern. Congress of Math., Kyoto, Japan (Math. Soc. Japan, Tokyo, 1990), p. 175.

42. Yu.L. Maistrenko and I.I. Stukanev, Dynamical Systems and Nonlinear Phenomena (Inst. of Math. of the NAS of Ukraine, Kyiv, 1990), p. 26 (in Russian).

43. Yu.K. Krasnev, in Nonlinear Waves. Structures and Bifurcations (Nauka, Moscow, 1987), p. 174 (in Russian).

44. E.A. Novikov, Zh. Eksp. Teor. Fiz. 68, 1868 (1975).

45. V.E. Zakharov and E.A. Kuznetsov, Zh. Eksp. Teor. Fiz. 91, 1310 (1986).

46. V.E. Zakharov, A.N. Pushkarev, and A.M. Rubenchik, Zh. Eksp. Teor. Fiz. 96, 591 (1989).

47. V.E. Zakharov, in Problems of Physical Kinetics and Physics of Solids (Naukova Dumka, Kiev, 1990), p. 469 (in Russian).
48. A.Y. Wong and P.Y. Cheng, Phys. Rev. Lett. 52, 1222 (1984).

49. A.C. Newell, D.A. Rand, and D. Russell, Physica D 33, No. 1-3, 281 (1988).

50. V.P. Maslov, Teor. Mat. Fiz. 69, 361 (1986).

51. V.P. Maslov, in Modern Problems of Applied Mechanics and Mathematical Physics (Nauka, Moscow, 1988), p. 60 (in Russian).

52. V.V. Kadomtsev, A.B. Mikhaylovskiy, and A.V. Timofeev, Zh. Eksp. Teor. Fiz. 17, 2266 (1964).

53. C. Boldrighini and N. Franceschini, Commun. Math. Phys. 64, 159 (1979).

54. N. Franceschini and C. Tebaldy, J. of Stat. Phys. 25, 397 (1981).

55. N.N. Brushlinskaya, in Some Questions of the Mechanics of Rocks, edited by L.V. Ershov (MGI, Moscow, 1968), p. 57 (in Russian).

56. S. Gonchenko, D. Turaev, and L. Silnikov, Physica D 62, No. 1-4, 1 (1993).

Received 15.07.11

\section{МОДЕЛЬНІ РІВНЯННЯ І УТВОРЕННЯ СТРУКТУР У СЕРЕДОВИЩАХ $З$ ПАМ'ЯТТЮ}

О.С. Макаренко

Р е $з$ ю м е

Наведено дослідження появи структур малого і великого масштабів у середовищах з пам'яттю. Запропоновано приклади нових типів моделей. Розглянуто гіперболічну модифікацію рівнянь Навье-Стокса та клас динамічних маловимірних моделей, які враховують ефекти пам'яті. Описано результати комп'ютерного моделювання, які ілюструють утворення структур малого масштабу і появу колапсів. Розглянуто появу нових хаотичних рішень в деяких моделях. Обговорено можливості застосування деяких запропонованих моделей до опису спалахів і виникнення колапсів на Сонці. 